\title{
KRAS genotyping in rectal adenocarcinoma specimens with low tumor cellularity after neoadjuvant treatment
}

Florence Boissière-Michot ${ }^{1, *}$, Evelyne Lopez-Crapez ${ }^{2, *}$, Hélène Frugier ${ }^{1}$, Marie-Laurence Berthe ${ }^{3}$, Alexandre Ho-Pun-Cheung ${ }^{2}$, Eric Assenat ${ }^{4}$, Thierry Maudelonde ${ }^{3}$, Pierre-Jean Lamy ${ }^{2}$ and Frédéric Bibeau ${ }^{1}$

${ }^{1}$ Department of Pathology, Val d'Aurelle Cancer Institute, Montpellier, France; ${ }^{2}$ Department of Biology, Val d'Aurelle Cancer Institute, Montpellier, France; ${ }^{3}$ Department of Cellular Biology and Hormonology, Arnaud de Villeneuve Hospital, Montpellier, France and ${ }^{4}$ Department of Medical Oncology,

Val d'Aurelle Cancer Institute, Montpellier, France

\begin{abstract}
KRAS status assessment is mandatory in patients with metastatic colorectal cancer before therapy with antiepidermal growth factor receptor monoclonal antibodies, as KRAS mutations are associated with resistance to this treatment. However, KRAS genotyping may be very challenging in case of poor tumor cellularity, particularly when major tumor regression is achieved in locally advanced rectal adenocarcinomas after radiochemotherapy. We aimed at identifying the most reliable strategy to detect KRAS mutations in such samples. DNA was extracted from 31 surgical specimens with major tumor regression, following manual dissection, and from paired pre-treatment biopsies and analyzed by high-resolution melting. DNA samples displaying altered melting curve shapes were then sequenced. Samples with unmodified melting curves or wild-type sequence were further investigated by using an allele-specific PCR assay (TheraScreen) and laser microdissection (followed by high-resolution melting and sequencing analyses). In the 31 post-radiochemotherapy surgical specimens, seven KRAS mutations were identified by high-resolution melting analysis/sequencing. One additional mutation was detected by the TheraScreen assay and two mutations, including the one identified by the TheraScreen assay, were detected following laser microdissection. Altogether, 9/31 surgical specimens $(29 \%)$ presented KRAS mutations. In the manually dissected pre-treatment biopsies, 12 mutations (39\%) were identified by high-resolution melting analysis and sequencing. No additional mutations were found by using the TheraScreen assay or laser microdissection. These results indicate that, in the case of post-radiochemotherapy surgical specimens of colorectal cancer with low tumor cellularity, pre-treatment biopsies might represent the most cost-effective option for reliable KRAS genotyping. The use of more sensitive assays, such as allelespecific PCR or laser microdissection, can be envisaged but with higher costs and longer delays. Modern Pathology (2012) 25, 731-739; doi:10.1038/modpathol.2011.210; published online 27 January 2012
\end{abstract}

Keywords: KRAS; mutation; neoadjuvant treatment; rectal cancer

Somatic mutations of the KRAS oncogene are present in $\sim 40 \%$ of colorectal tumors and are a reliable marker of resistance to therapy with monoclonal

Correspondence: Dr F Bibeau, MD, PhD, Service de Pathologie, CRLC Val d'Aurelle, 208 rue des Apothicaires, Montpellier Cedex 5 34298, France.

E-mail: Frederic.Bibeau@montpellier.unicancer.fr

This study was presented in part at the USCAP 100th annual meeting, 26 February to 4 March 2011; San Antonio, Texas.

*These authors contributed equally to this work.

Received 15 September 2011; revised 7 November 2011; accepted

9 November 2011; published online 27 January 2012 antibodies (cetuximab or panitumumab) that target epidermal growth factor receptor (EGFR).$^{1-7}$ Indeed, KRAS mutations result in constitutively active KRAS proteins that lead to continuous activation of the RAS signaling pathway independently of the upstream stimulation by EGFR ligands. As only patients with wild-type KRAS tumors benefit from therapies with anti-EGFR antibodies, accurate determination of the KRAS mutation status is crucial for ethical and economic reasons.

To date, there is no standardized operating procedure for KRAS status determination in the clinical 
setting, although recommendations have been proposed. $^{8-11}$ Various laboratory-based or commercial KRAS mutation assays are used in the routine practice and the majority of them have been optimized to be compatible with DNA extracted from formalin-fixed paraffin-embedded samples, which represent the most available source of tumor tissue. However, they are characterized by different sensitivity, turnaround time, and cost. ${ }^{12-15}$

Besides the use of robust KRAS testing methods, the selection of the appropriate tissue to be tested is also critical. Particularly, evaluation of the tumor cellularity is essential to guide the interpretation of the molecular data. For instance, false-negative results can occur when the starting sample is contaminated by an important number of non-tumor cells. ${ }^{16-18}$ In the case of metastatic colorectal cancer, poor tumor cellularity is mainly observed when major tumor regression is achieved in locally advanced rectal adenocarcinomas after radiochemotherapy or in liver metastases after chemotherapy.10,19,20 Manual dissection to remove the non-invasive cell components before nucleic acid extraction is a powerful tool for tumor cell enrichment. However, the manual dissection of some specimens with scattered tumor cells, especially after neoadjuvant treatment, or with many inflammatory cells can be challenging. One way to circumvent this issue would be to perform laser capture microdissection. ${ }^{17,21}$

The goal of this study was to determine the most reliable combination of starting material and molecular testing procedures to detect KRAS mutations in challenging cancer samples with poor tumor cellularity. Using a prospective series of samples from patients with locally advanced rectal cancer treated by neoadjuvant radiochemotherapy, we compared the usefulness of laser capture microdissection and manual dissection and three KRAS mutation detection assays with different ranges of sensitivity in pre-therapeutic biopsies and paired adenocarcinomas resected after neoadjuvant radiochemotherapy.

\section{Materials and methods}

The experimental protocol of this study is summarized in Figure 1.

\section{Patients and Tissue Samples}

The specimens analyzed in this study were selected from a previously described prospective series. ${ }^{22,23}$ Between November 2005 and May 2008, 71 patients with locally advanced, histologically confirmed rectal adenocarcinoma were recruited in a prospective study conducted at the Val d'Aurelle PaulLamarque Cancer Center in Montpellier. For all patients, informed consent for the use of clinical records and tumor specimens for research purposes was obtained. The protocol was approved by the Saint-Eloi Hospital Ethics Committee (Montpellier,

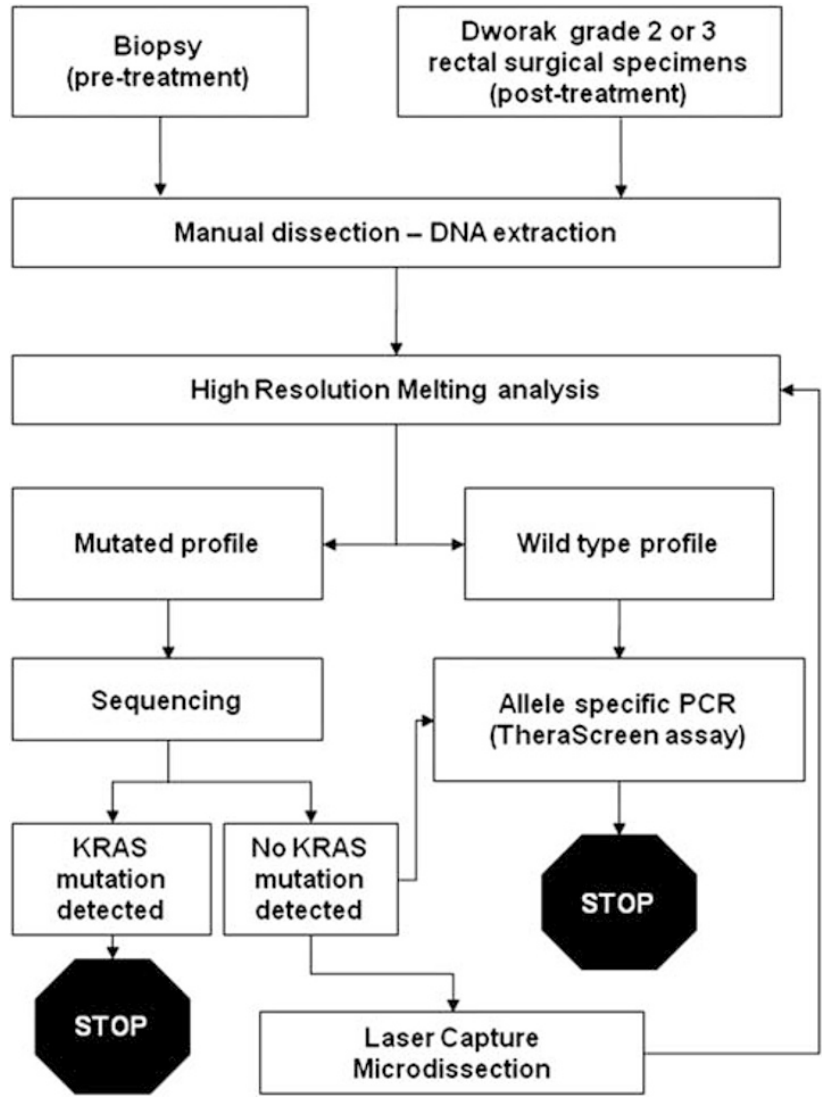

Figure 1 Strategy used to evaluate the usefulness of laser microdissection (vs manual dissection) and the combination of three KRAS mutation detection assays including high-resolution melting, ARMS/Scorpion assay and direct sequencing.

France) for the protection of patients involved in biomedical research.

For all patients, two pre-treatment biopsies were obtained by endoscopy. They were immediately fixed in AFA (ethanol + formol + acetic acid) for $12-24 \mathrm{~h}$ and then embedded in paraffin. The biopsy with the highest amount of tumor cells was selected for this study. All patients underwent preoperative radiotherapy with concurrent chemotherapy (capecitabine or capecitabine + oxaliplatin). Surgery was scheduled 6-8 weeks after the completion of the preoperative radiochemotherapy. Surgical specimens were fixed in formalin for $48 \mathrm{~h}$. The whole macroscopic tumor area was isolated and paraffin embedded. Sections were stained with hematoxylin, eosin, and saffron (HES) for histological examination. The tumor response to preoperative radiochemotherapy was semiquantitatively determined according to the tumor regression grading system proposed by Dworak et al. ${ }^{24}$ Grading ranged from 0 (no evidence of any treatment effect) to 4 (complete response with no viable tumor cells). Grade 1 corresponded to a dominant tumor mass with obvious fibrosis or mucin, grade 2 to dominantly fibrotic or mucinous changes with few tumor cells or groups, and grade 3 to specimens with very few tumor cells 


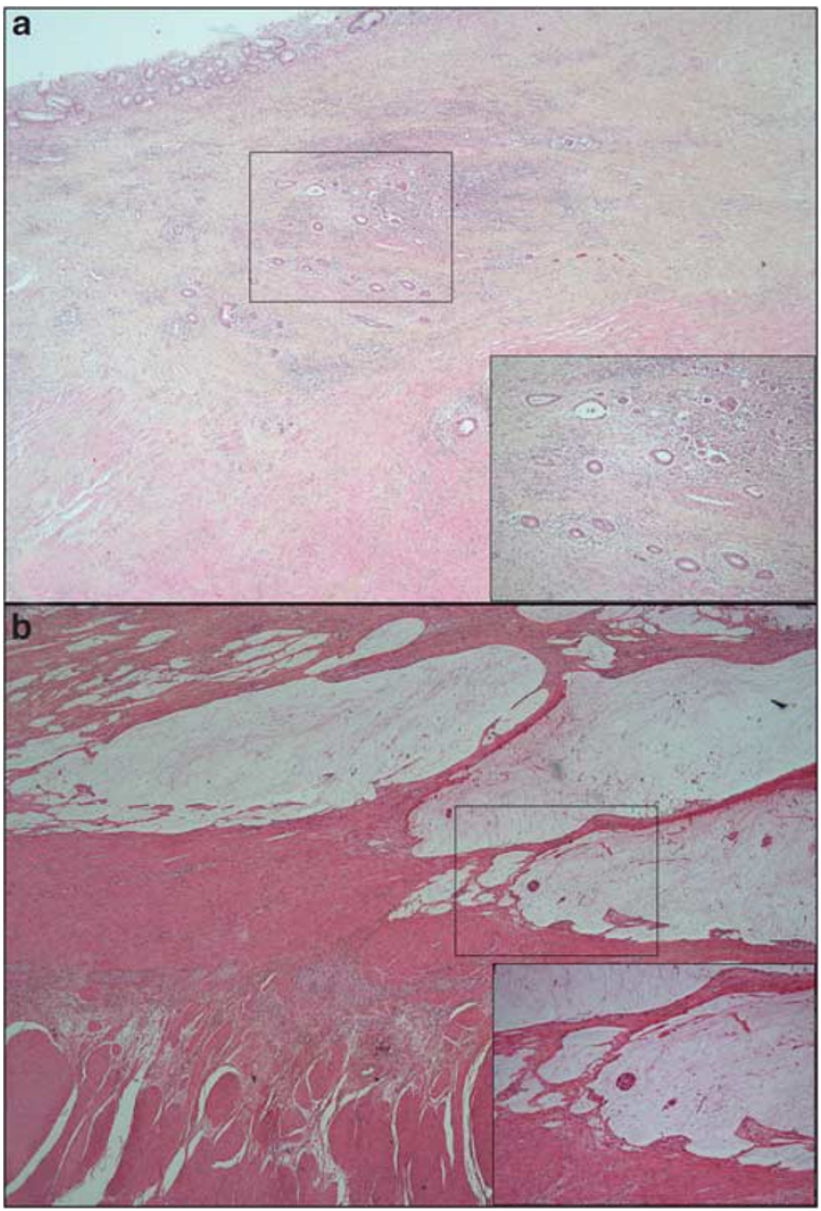

Figure 2 Dworak grade 2 (a) and 3 (b) rectal adenocarcinomas removed after neoadjuvant radiochemotherapy. The malignant component (insert) is scattered within fibrotic tissue (a) or mucin (b).

in fibrotic tissue with or without mucous substance. Only grade $2(n=15$; Figure $2 \mathrm{a})$ and grade $3(n=16$; Figure 2b) specimens, that is, with few or very few tumors cells, were selected for this study.

\section{Manual Dissection and Microdissection of Tissue Samples}

HES-stained sections from pre-treatment biopsies and post-radiochemotherapy samples were used to select tumor areas for manual dissection. The regions of interest, that is, those containing tumor cells, were located under a microscope and surrounded with a pen. Ten consecutive adjacent $5 \mu \mathrm{m}$-thin sections were then manually dissected by removing the surrounding non-neoplastic components in the selected areas. An additional HESstained section was systematically taken to confirm the presence of tumor cells. HES-stained sections were reviewed by two trained observers to assess the percentage of tumor cells in the selected area. Samples were then ranked in three classes: $<20 \%$, between 20 and $50 \%$, and $>50 \%$ of tumor cells in the dissected area.

If laser capture microdissection was required (ie if no mutation was identified after high-resolution melting and sequencing; see Figure 1), 3-10 additional $7 \mu \mathrm{m}$-thin serial sections were used. Laser capture microdissection was performed using the PixCell II LCM System and CapSure HS LCM caps (Arcturus Engineering, Mountain View, CA, USA). Tissue sections were deparaffinized before staining with hematoxylin-eosin. Slides were then stored in a desiccated container for at least $15 \mathrm{~min}$ before laser capture microdissection. All distinguishable tumor cells or glands present in the studied sections were microdissected (Figure 3). Laser capture microdissection efficiency was assessed by examining the cells present in the cap. Caps were placed in $500 \mu \mathrm{l}$ microcentrifuge tubes and kept in a box at room temperature until DNA extraction.

\section{DNA Extraction}

DNA was extracted using the QIAamp DNA FFPE (manually dissected samples) or the QIAamp DNA micro Kit (laser capture microdissected samples) (Qiagen, Hilden, Germany) according to the manufacturer's protocols. Only DNA extracted from manually dissected specimens was quantified using a NanoDrop spectrophotometer (Thermo Scientific, Wilmington, USA), because DNA extraction from laser capture microdissected samples required a carrier that absorbs at $260 \mathrm{~nm}$, thus preventing adequate quantification.

\section{KRAS Exon 2 Mutation Analysis}

\section{High-resolution melting analysis}

For high-resolution melting screening, an 84-bp fragment from KRAS exon 2 (primers available upon request) was PCR amplified using a Rotor-Gene 6000 instrument (Qiagen) and the LightCycler 480 High-Resolution Melting Master Reaction Mix (Roche Diagnostics, Meylan, France). Reactions were carried out in $20 \mu \mathrm{l}$ total volume that included $25 \mathrm{ng}$ purified genomic DNA, $10 \mu \mathrm{l}$ of $2 \times$ PCR reaction mix, $3.0 \mathrm{mM} \mathrm{MgCl}_{2}$ and $0.25 \mu \mathrm{M}$ of each forward and reverse primer. The cycling conditions were as follows: one cycle of $95{ }^{\circ} \mathrm{C}$ for $5 \mathrm{~min}$, followed by 50 cycles at $95^{\circ} \mathrm{C}$ for $15 \mathrm{~s}, 63^{\circ} \mathrm{C}$ for $25 \mathrm{~s}$ with a touchdown program of $0.5^{\circ} \mathrm{C} / \mathrm{cycle}$ during the first 11 cycles, and $72{ }^{\circ} \mathrm{C}$ for $25 \mathrm{~s}$. The melting conditions included one cycle of $95^{\circ} \mathrm{C}$ for $1 \mathrm{~min}$, one cycle of $40^{\circ} \mathrm{C}$ for $1 \mathrm{~min}$, and one cycle of $65^{\circ} \mathrm{C}$ for $2 \mathrm{~s}$, followed by a melt from $65^{\circ} \mathrm{C}$ to $95^{\circ} \mathrm{C}$ rising at a rate of $0.1^{\circ} \mathrm{C}$ per second. All samples were tested in duplicate. High-resolution melting data were analyzed using the Rotor-Gene 6000 software (v1.7). For each sample, normalized melting curves were evaluated, and the studied samples were compared with wild-type controls, consisting of genomic 

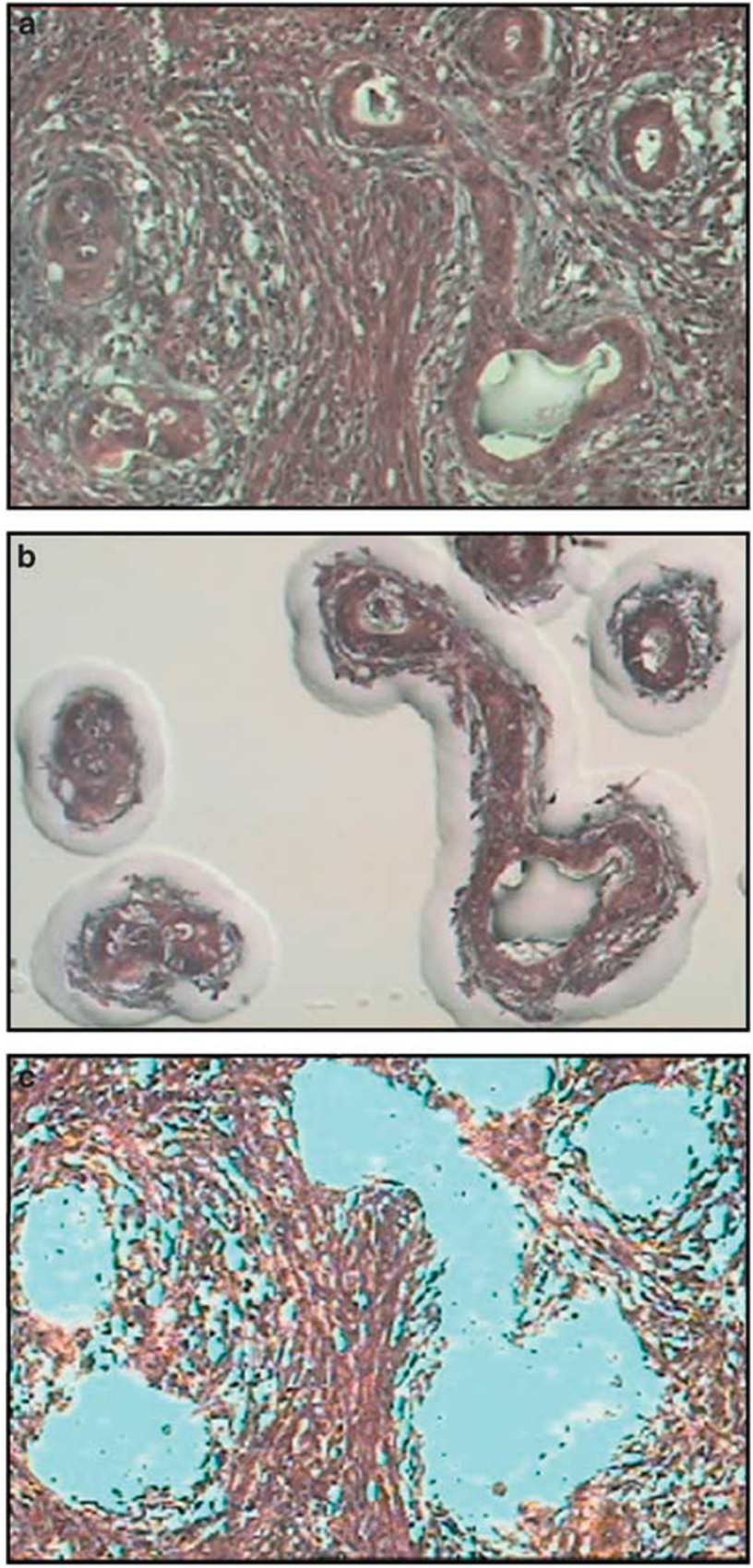

Figure 3 Dworak grade 2 rectal adenocarcinoma stained with hematoxylin-eosin (a). The malignant glands were isolated by laser capture microdissection and visualized in the cap (b), leaving the stromal component on the slide (c).

DNA extracted from normal rectal tissues and processed like the specimens of interest in a deduced difference plot.

\section{Sequencing}

Bidirectional DNA sequencing for detection of KRAS exon 2 mutations was performed by using two seminested amplicons: one of $245 \mathrm{bp}$ covering the entire KRAS exon 2 region, as previously described, ${ }^{25}$ and a shorter one of $164 \mathrm{bp}$. Primers used for amplification and sequencing and PCR conditions are available upon request. Direct sequencing was done using the BigDye Terminator v1.1 Cycle Sequencing Kit (Applied Biosystems, Courtaboeuf, France) before electrophoresis on a 3130 Genetic analyzer (Applied Biosystems). KRAS sequences were then analyzed with the Applied Biosystems SeqScape software v2.5. All samples were analyzed in duplicate by direct sequencing and mutations were confirmed by sequencing independent PCR products.

\section{Allele-specific PCR}

The commercially available TheraScreen KRAS kit (DxS Diagnostics, Manchester, UK) based on the ARMS and Scorpions technologies detects the seven most common KRAS exon 2 mutations by realtime quantitative PCR. The test identifies six mutations in codon 12 (p.G12A, p.G12D, p.G12R, p.G12C, p.G12S, and p.G12V) and one in codon 13 (p.G13D). Mutation analysis was performed following the manufacturer's instructions on a Rotor-Gene 6000 instrument (Qiagen). For each real-time PCR run, a positive reaction mix containing the seven KRAS exon 2 mutations was included. Each sample was tested for a total of eight amplifications: seven mutation reactions and one control reaction corresponding to the amplification of part of KRAS exon 4. Moreover, for each KRAS-specific amplification, a second exogenous reaction was simultaneously performed, to avoid false-negative results caused by PCR inhibitors. Then, the cycle threshold (Ct) for each KRAS reaction was determined. Samples with a Ct value of $\geq 35$ in the control assay were rejected. Samples were considered to carry a mutation only when the $\Delta \mathrm{Ct}$ (Ct of control reaction-Ct mutation reaction) value was lower than the value set by the manufacturer.

\section{Results}

\section{Contribution of Manual Dissection}

To ensure the best ratio of tumor cells to non-tumor cells, all the post-radiochemotherapy surgical specimens and the paired pre-treatment biopsies were manually dissected. Despite a careful selection of the tumor areas, only $13 \%(4 / 31)$ of the surgical specimens displayed $>50 \%$ of tumor cells in the dissected areas and $68 \%(21 / 31)$ had $<20 \%$ of tumor cells. Conversely, $55 \%$ (17/31) of the pretreatment biopsies had $>50 \%$ of tumor cells and only $19 \%(6 / 31)$ had $<20 \%$ of tumor cells (Table 1 ).

\section{KRAS Mutation Status of the Pre-Treatment Biopsies}

Manual dissection of pre-treatment biopsies allowed the recovery of $1.20 \pm 0.85 \mu \mathrm{g}$ of DNA (mean \pm standard error of the mean; $\min : 0.15 \mu \mathrm{g}$; max: $3.97 \mu \mathrm{g}$ ) and the completion of all planned analyses. 
In 2010, KRAS mutation testing in our institute routinely combined manual dissection and highresolution melting analysis followed by sequencing in case of abnormal profile. ${ }^{26}$ This strategy allowed the identification of KRAS mutations in $12 / 31$ pretreatment biopsies (39\%, 6 Dworak grade 2 and 6 Dworak grade 3). The identified KRAS mutations (and their frequency) were G12D (3/12), G12V (1/12), G12C (2/12), G12S (2/12), G12R (1/12), G13D (1/12), G13C (1/12), and G13R (1/12). The G12A mutation was not detected in this study. Confirmation of the two uncommon KRAS codon 13 mutations (G13C and G13R) was performed by pyrosequencing (data not shown). No additional mutations were found after analysis of the pre-treatment biopsies with a normal high-resolution melting profile or wild-type KRAS status (by bidirectional sequencing) by using the TheraScreen assay or laser capture microdissection.

\section{KRAS Mutation Status of the Post-Radiochemotherapy Rectal Surgical Specimens}

Manual dissection of the 31 surgical specimens allowed the recovery of $4.46 \pm 4.34 \mu \mathrm{g}$ DNA (mean \pm s.e.m.; min: $0.63 \mu \mathrm{g}$; max: $17.03 \mu \mathrm{g}$ ).

Table 1 Assessment of tumor cell content in pre-treatment biopsies and paired post-radiochemotherapy surgical specimens after manual microdissection; number and (percentage)

\begin{tabular}{lcc}
\hline $\begin{array}{l}\% \text { of tumor cells } \\
\text { relative to } \\
\text { non-tumor cells }\end{array}$ & $\begin{array}{c}\text { Pre-treatment } \\
\text { rectal biopsies }\end{array}$ & $\begin{array}{c}\text { Post-radiochemotherapy } \\
\text { rectal surgical } \\
\text { specimens }\end{array}$ \\
\hline$<20 \%$ & $6(19 \%)$ & $21(68 \%)$ \\
$20-50 \%$ & $8(26 \%)$ & $6(19 \%)$ \\
$>50 \%$ & $17(55 \%)$ & $4(13 \%)$ \\
\hline
\end{tabular}

Using the same routine procedure as for pretreatment biopsies (high-resolution melting analysis and sequencing), KRAS mutations were identified in 7/31 (23\%) post-radiochemotherapy surgery specimens of rectal adenocarcinoma (G12D: 2; G12C: 1; G12S: 2; G12R: 1; and G13D: 1), among which 6 Dworak grade 2 and 1 Dworak grade 3 . The 24 tumors in which no mutation was detected were further analyzed by using the TheraScreen KRAS Mutation kit that identified one additional G12D mutation. Moreover, laser capture microdissection followed by high-resolution melting and sequencing allowed the detection of two additional mutations: the G12D mutation identified by the TheraScreen assay (Figure 4; Patient A) and one G13C mutation (Figure 4b; Patient B). Altogether, 9/31 surgical specimens $(29 \%)$ carried KRAS mutations.

Importantly, each mutation detected in postradiochemotherapy surgical samples was also observed in the corresponding pre-treatment biopsy specimen.

\section{Discussion}

KRAS mutation testing is mandatory for the identification of patients with metastatic colorectal cancer who may benefit from treatment with anti-EGFR monoclonal antibodies. However, the best way to determine the KRAS status is still a matter of debate, particularly for samples with low tumor cellularity. The aim of our work was to determine the most reliable combination of starting material and testing methodologies to detect KRAS mutations in rectal adenocarcinoma specimens with important tumor regression following neoadjuvant radiochemotherapy. Comparison of the results obtained in pre-treatment biopsies and post-radiochemotherapy samples using
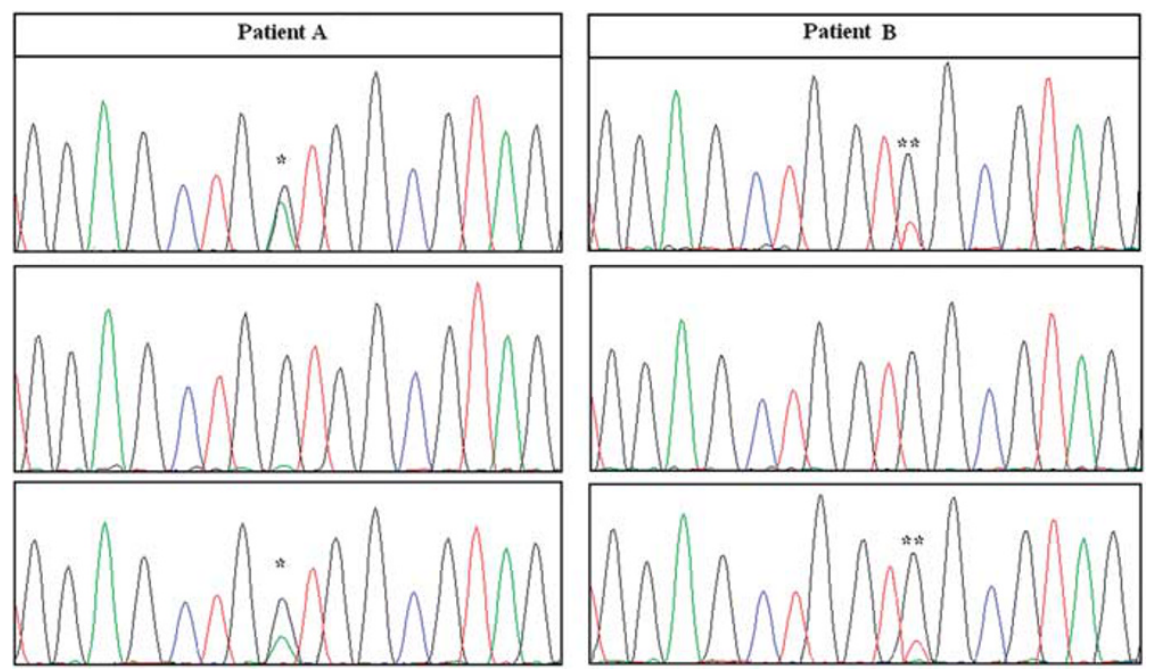

Figure 4 Sanger sequencing of KRAS exon 2 in DNA isolated from manually dissected pre-treatment biopsies (top), manually dissected (middle) or laser microdissected surgical specimens (bottom) of two rectal adenocarcinomas. A p.G12D $\left({ }^{*}\right)$ mutation $($ Patient A, left panels) and a p.G13C $\left({ }^{* *}\right)$ mutation (Patient B, right panels) were identified in the pre-treatment biopsies and in the laser microdissected surgical specimens. Manual dissection of the surgical specimens did not allow the detection of any KRAS exon 2 mutation. 
manual dissection or laser capture microdissection and three KRAS mutation detection assays indicates that, in our hands, pre-treatment biopsies and highresolution melting analysis followed by direct sequencing represent the most cost-effective option.

In the routine diagnostic setting, several issues should be considered when performing KRAS genotyping to guide proper treatment selection. First, the genetic heterogeneity of the starting material must be taken into account. Particularly, since the proportion of tumor and non-tumor cells in specimens is highly variable, the percentage of mutant DNA in the DNA isolated from such samples will also be variable. We and others have previously demonstrated the inverse relationship between tumor cell content of a specimen and ability to detect mutations by direct sequencing. ${ }^{16,18,19,27}$ Thus, the method used to detect somatic mutations must be sensitive enough to avoid false-negative results, particularly in samples contaminated by non-tumor elements.

A way to increase sensitivity is to limit the dilution of tumor DNA in the background of normal DNA by performing tumor cell enrichment before DNA extraction as recommended in the technical guidelines by the European Society of Pathology and the College of American Pathologists. ${ }^{11,28}$ Manual dissection of formalin-fixed paraffin-embedded tissues is a widely used and powerful tool to enrich the sample with tumor cells. ${ }^{17,27}$ This pre-analytical step is easy to perform, not time consuming and suitable for a routine setting. However, the work by Plesec and Hunt $^{10}$ and our study show that manual dissection is insufficient for some samples, such as rectal surgical specimens with a significant tumor regression after radiochemotherapy. Interestingly, in our study, the five discordant post-radiochemotherapy samples were all graded as Dworak 3, that is, with very few tumor cells in fibrotic tissue or mucous substance, suggesting that tumor cell content is the cornerstone of a reliable mutational analysis. The use of core biopsies in combination with sequencing or allele-specific PCR increases the frequency of detection of KRAS mutations in comparison with whole tissue sections. ${ }^{29}$ However, the issue of samples with low tumor cellularity was not specifically addressed with this technique. Another way to increase tumor cell enrichment is to perform a more selective dissection of the scattered tumor cells/glands by laser capture microdissection. ${ }^{17,30}$ In our hands, laser capture microdissection is efficient (two additional mutations were identified), but does not seem to be suitable for a routine setting because it is time- and laborconsuming and, therefore, expensive. In practice, 10-100 min were necessary for microdissecting a sample, depending on its tumor cellularity. Moreover, its availability only in a small number of laboratories precludes its wide use.

Another way to decrease the risk of false wildtype KRAS status in samples with low tumor cellularity would be to use more sensitive KRAS genotyping methods. Many assays with variable sensitivity, specificity, and costs are currently available (reviewed in van Krieken et $a l^{11}$ ). Due to its exhaustiveness, the multi-step sequencing methodology is the gold standard for the detection of unknown mutations, but it lacks sensitivity. Consequently, more rapid and sensitive methods have been developed and validated, including highresolution melting, ${ }^{29,31}$ allele-specific PCR, ${ }^{16,29}$ and pyrosequencing. ${ }^{32}$ Performed before DNA sequencing, high-resolution melting is very attractive because the analysis of the real-time amplification curves allows (i) the calculation of the Ct value that is directly related to the amount of amplifiable DNA and (ii) the identification of heterozygous genetic changes even in samples containing only $10 \%$ of mutated cells. ${ }^{33}$ In our series of DNA extracted from the manually dissected specimens, all Ct values were $<30$, whereas higher values were obtained for the paired laser capture microdissected samples (data not shown), suggesting a negative impact of laser capture microdissection on DNA. Concerning the allele-specific PCR assay, the first kit approved for diagnostic purposes was the TheraScreen kit that can detect as few as $1 \%$ of mutated alleles in a wild-type background and is a simple 'mix-andmeasure' test. ${ }^{34}$ Nevertheless, compared with test based on end point PCR, this real-time PCR approach is more sensitive to potential PCR inhibitors and DNA quality. Moreover, as eight real-time PCR reactions are performed for each DNA to study, the starting amount of material required for mutational status determination may be a limitation. In our series, high-resolution melting, direct sequencing and TheraScreen analysis (when required) were performed for all manually dissected samples. In previous studies, a failure rate of $5-10 \%$ was reported for the TheraScreen technology ${ }^{32}$ in relation to limited or poor quality material. ${ }^{35,36}$ In our hands, the TheraScreen assay allowed the detection of only one additional mutation in postradiochemotherapy samples that had a mutated high-resolution melting profile but was identified as wild type by direct sequencing. Pre-treatment biopsies, which had higher tumor cell content, did not benefit from this technology. Moreover, if only the TheraScreen assay had been used instead of direct sequencing, 2/12 identified mutations (G13C, G13R) would have been missed, as they do not belong to the panel of mutations targeted by the designed primers. This illustrates the major pitfall of such allele-specific PCR assays.

Another issue to be considered when testing for KRAS mutations is the occurrence of artifactual mutations. Unreliable genotyping can be generated when the starting amount of DNA is low. ${ }^{37}$ Artifactual mutations can be observed if nucleotide misincorporation occurs during the first PCR cycle $^{38}$ or if chemical modifications have been induced by formalin fixation. ${ }^{39-41}$ To reduce such artifacts, it is recommended to use a minimum of 
$1 \mu \mathrm{g}$ of formalin-fixed paraffin-embedded-recovered DNA and to confirm each identified mutation by an independent analysis. ${ }^{38,42}$ In our practice, manual dissection of pre-treatment biopsies allowed the recovery of an amount of DNA that was enough for our study protocol. Unlike Lamy et $a l^{42}$ we did not observe artifactual mutations, even when using small amounts of template DNA. Indeed, when identified, the KRAS mutation was the same in the paired biopsy and surgical specimen, whatever the molecular assay used. The small number of patients and the homogeneous tissue processing might have contributed to the absence of artifacts in our study. In the same way, Ondrejka et $a l^{43}$ recently reported concordant mutation results in 17 rectal specimens before and after radiochemotherapy. However, their study was designed to address the question of the impact of neoadjuvant treatment on DNA mutation but was not focused on the putative risk of false wild-type tumors linked to poor cellularity. When using laser capture microdissection, minimal amounts of fragmented DNA are obtained and this may restrict the choice of genetic assays. To overcome this limitation, whole genome amplification may represent an attractive option ${ }^{44}$ because it does not introduce genotypic changes and might allow the assessment of any potentially mutated region in the genome. ${ }^{15}$

Finally, the possible impact of the sensitivity threshold of the KRAS mutation assays should also be considered. Recently, two groups ${ }^{45,46}$ reported that the use of highly sensitive assays (allele-specific PCR with probe inhibiting the amplification of wildtype sequences and pyrosequencing) allowed, as expected, the retrospective identification of additional KRAS mutations in a population of patients previously identified as wild type. Interestingly, among the seven patients with a KRAS mutation detected with these highly sensitive techniques and who received cetuximab-based chemotherapy, five were responders. This finding suggests that the use of very sensitive technologies for KRAS genotyping in the diagnostic setting could lead to exclusion of patients who may benefit from anti-EGFR therapy. Thus, they should be restricted to the analysis of samples with a low proportion of tumor cells. Pyrosequencing-based techniques, such as standard pyrosequencing ${ }^{47}$ or pyrosequencing on high-density picoliter plates using the Roche 454 system, ${ }^{48}$ allow the determination not only of the mutation but also of the proportion of mutant and wild-type sequences in each sample. It would thus be interesting to determine the threshold below which KRAS mutations do not negatively influence the tumor response to anti-EGFR therapy. For this, the standardization of the pre-analytic phase is required, especially the assessment of the proportion of tumor cells present in the sample used for DNA extraction.

In summary, in this work we investigated the best KRAS mutation testing strategy for samples with low amount of tumor cells. Using rectal post-radiochemotherapy surgical specimens and paired pretreatment biopsies, we show that pre-treatment biopsies represent the best starting material for mutation detection. Thus, we recommend performing a tumor pre-treatment biopsy dedicated to molecular diagnosis before any neoadjuvant treatment. However, if not available, the use of more sensitive assays could help decreasing the risk of false negative (in our series, $25 \%$, that is, $3 / 12$ identified mutated samples). Although laser capture microdissection allowed the identification of additional mutations, it does not appear suitable for a routine setting due to the time required. On the other side, the TheraScreen technology based on allele-specific PCR allows the detection of only a restricted set of common mutations.

To accurately interpret the KRAS testing results for a patient-tailored therapeutic choice, data from the pre-analytical and analytical steps must be considered jointly. Particularly, rigorous quality control and morphologic review of the starting tissues are required to select the more appropriate KRAS genotyping test.

\section{Acknowledgements}

The laboratories involved in this study are all part of the 'Génétique Moléculaire des Tumeurs' facility of the Languedoc-Roussillon region and funded by the French Institut National du Cancer (INCa). Part of this study was funded by Amgen.

\section{Disclosure/conflict of interest}

The authors declare no conflict of interest.

\section{References}

1 Amado RG, Wolf M, Peeters M, et al. Wild-type KRAS is required for panitumumab efficacy in patients with metastatic colorectal cancer. J Clin Oncol 2008;26:1626-1634.

2 De Roock W, Piessevaux H, De Schutter J, et al. KRAS wild-type state predicts survival and is associated to early radiological response in metastatic colorectal cancer treated with cetuximab. Ann Oncol 2008;19: 508-515.

3 Di Fiore F, Blanchard F, Charbonnier F, et al. Clinical relevance of KRAS mutation detection in metastatic colorectal cancer treated by Cetuximab plus chemotherapy. Br J Cancer 2007;96:1166-1169.

4 Karapetis CS, Khambata-Ford S, Jonker DJ, et al. K-ras mutations and benefit from cetuximab in advanced colorectal cancer. N Engl J Med 2008;359:1757-1765.

5 Khambata-Ford S, Garrett CR, Meropol NJ, et al. Expression of epiregulin and amphiregulin and K-ras mutation status predict disease control in metastatic colorectal cancer patients treated with cetuximab. J Clin Oncol 2007;25:3230-3237. 
6 Lievre A, Bachet JB, Le Corre D, et al. KRAS mutation status is predictive of response to cetuximab therapy in colorectal cancer. Cancer Res 2006;66:3992-3995.

7 Lievre A, Bachet JB, Boige V, et al. KRAS mutations as an independent prognostic factor in patients with advanced colorectal cancer treated with cetuximab. J Clin Oncol 2008;26:374-379.

8 Allegra CJ, Jessup JM, Somerfield MR, et al. American Society of Clinical Oncology provisional clinical opinion: testing for KRAS gene mutations in patients with metastatic colorectal carcinoma to predict response to anti-epidermal growth factor receptor monoclonal antibody therapy. J Clin Oncol 2009;27:2091-2096.

9 Bellon E, Ligtenberg MJ, Tejpar S, et al. External quality assessment for KRAS testing is needed: setup of a European program and report of the first joined regional quality assessment rounds. Oncologist 2011;16:467-478.

10 Plesec TP, Hunt JL. KRAS mutation testing in colorectal cancer. Adv Anat Pathol 2009;16:196-203.

11 van Krieken JH, Jung A, Kirchner T, et al. KRAS mutation testing for predicting response to anti-EGFR therapy for colorectal carcinoma: proposal for an European quality assurance program. Virchows Arch 2008;453:417-431.

12 Jimeno A, Messersmith WA, Hirsch FR, et al. KRAS mutations and sensitivity to epidermal growth factor receptor inhibitors in colorectal cancer: practical application of patient selection. J Clin Oncol 2009; 27:1130-1136.

13 Kobunai T, Watanabe T, Yamamoto Y, Eshima K. The frequency of KRAS mutation detection in human colon carcinoma is influenced by the sensitivity of assay methodology: a comparison between direct sequencing and real-time PCR. Biochem Biophys Res Commun 2010;395:158-162.

14 Oliner K, Juan T, Suggs S, et al. A comparability study of 5 commercial KRAS tests. Diagn Pathol 2010;16:5-23.

15 van Eijk R, van Puijenbroek M, Chhatta AR, et al. Sensitive and specific KRAS somatic mutation analysis on whole-genome amplified DNA from archival tissues. J Mol Diagn 2010;12:27-34.

16 Angulo B, Garcia-Garcia E, Martinez R, et al. A commercial real-time PCR kit provides greater sensitivity than direct sequencing to detect KRAS mutations: a morphology-based approach in colorectal carcinoma. J Mol Diagn 2010;12:292-299.

17 Hunt JL, Finkelstein SD. Microdissection techniques for molecular testing in surgical pathology. Arch Pathol Lab Med 2004;128:1372-1378.

18 Tol J, Dijkstra JR, Vink-Borger ME, et al. High sensitivity of both sequencing and real-time PCR analysis of KRAS mutations in colorectal cancer tissue. J Cell Mol Med 2009;14:2122-2131.

19 Bibeau F, Frugier H, Crapez E, et al. KRAS mutation testing in patients with metastatic colorectal carcinoma: an 18-month experience emphasizing the risk of false-negative results. J Clin Oncol 2010;28:15s (Suppl; abstr 3572).

20 Selves J, Grand D, Guimbaud R, et al. Impact of the amount of tumor cells in tissue samples for detection of KRAS mutations in colorectal cancer. J Clin Oncol 2010;28:15s (Suppl; abstr 3571).

21 Danjoux M, Guimbaud R, Al Saati T, et al. Contribution of microdissection for the detection of microsatellite instability in colorectal cancer. Hum Pathol 2006;37:361-368.
22 Ho-Pun-Cheung A, Assenat E, Bascoul-Mollevi C, et al. A large-scale candidate gene approach identifies SNPs in SOD2 and IL13 as predictive markers of response to preoperative chemoradiation in rectal cancer. Pharmacogenomics J 2011;11:437-443.

23 Ho-Pun-Cheung A, Assenat E, Bascoul-Mollevi C, et al. EGFR and HER3 mRNA expression levels predict distant metastases in locally advanced rectal cancer. Int J Cancer 2011;128:2938-2946.

24 Dworak O, Keilholz L, Hoffmann A. Pathological features of rectal cancer after preoperative radiochemotherapy. Int J Colorectal Dis 1997;12:19-23.

25 Lopez-Crapez E, Chypre C, Saavedra J, et al. Rapid and large-scale method to detect K-ras gene mutations in tumor samples. Clin Chem 1997;43:936-942.

26 Lopez-Crapez E, Mineur L, Emptas H, Lamy PJ. KRAS status analysis and anti-EGFR therapies: is comprehensiveness a biologist's fancy or a clinical necessity? Br J Cancer 2010;102:1074-1075.

27 Weichert W, Schewe C, Lehmann A, et al. KRAS genotyping of paraffin-embedded colorectal cancer tissue in routine diagnostics: comparison of methods and impact of histology. J Mol Diagn 2010;12: 35-42.

28 Perspectives on Emerging Technology (POET). Report: KRAS Mutation Testing in Colorectal Cancer (CRCs). College of American Pathologists: Northfield, IL, 2009.

29 Franklin WA, Haney J, Sugita M, et al. KRAS mutation: comparison of testing methods and tissue sampling techniques in colon cancer. J Mol Diagn 2010;12: 43-50.

30 Bazan V, La Rocca G, Corsale S, et al. Laser pressure catapulting (LPC): optimization LPC-system and genotyping of colorectal carcinomas. J Cell Physiol 2005;202:503-509.

31 Ririe KM, Rasmussen RP, Wittwer CT. Product differentiation by analysis of DNA melting curves during the polymerase chain reaction. Anal Biochem 1997;245: 154-160.

32 Sundstrom M, Edlund K, Lindell M, et al. KRAS analysis in colorectal carcinoma: analytical aspects of Pyrosequencing and allele-specific PCR in clinical practice. BMC Cancer 2010;10:660.

33 Do H, Krypuy M, Mitchell PL, et al. High resolution melting analysis for rapid and sensitive EGFR and KRAS mutation detection in formalin fixed paraffin embedded biopsies. BMC Cancer 2008;8:142.

34 Cross J. DxS Ltd. Pharmacogenomics 2008;9:463-467.

35 Whitehall V, Tran K, Umapathy A, et al. A multicenter blinded study to evaluate KRAS mutation testing methodologies in the clinical setting. J Mol Diagn 2009;11:543-552.

36 Garm Spindler KL, Pallisgaard N, Rasmussen AA, et al. The importance of KRAS mutations and EGF61A $>\mathrm{G}$ polymorphism to the effect of cetuximab and irinotecan in metastatic colorectal cancer. Ann Oncol 2009;20:879-884.

37 Trusky CL, Sepulveda AR, Hunt JL. Assessment of microsatellite instability in very small microdissected samples and in tumor samples that are contaminated with normal DNA. Diagn Mol Pathol 2006;15:63-69.

38 Akbari M, Hansen MD, Halgunset J, et al. Low copy number DNA template can render polymerase chain reaction error prone in a sequence-dependent manner. J Mol Diagn 2005;7:36-39. 
39 Liu Y, Li CM, Lu Z, et al. Studies on formation and repair of formaldehyde-damaged DNA by detection of DNA-protein crosslinks and DNA breaks. Front Biosci 2006;1:991-997.

40 Srinivasan M, Sedmak D, Jewell S. Effect of fixatives and tissue processing on the content and integrity of nucleic acids. Am J Pathol 2002;161:1961-1971.

41 Williams C, Ponten F, Moberg C, et al. A high frequency of sequence alterations is due to formalin fixation of archival specimens. Am J Pathol 1999;155:1467-1471.

42 Lamy A, Blanchard F, Le Pessot F, et al. Metastatic colorectal cancer KRAS genotyping in routine practice: results and pitfalls. Mod Pathol 2011;24:1090-1100.

43 Ondrejka SL, Schaeffer DE, Jakubowski MA, et al. Does neoadjuvant therapy alter KRAS and/or MSI results in rectal adenocarcinoma testing? Am J Surg Pathol 2011; 35:1327-1330.
44 Hughes S, Arneson N, Done S, Squire J. The use of whole genome amplification in the study of human disease. Prog Biophys Mol Biol 2005;88: 173-189.

45 Santini D, Galluzzo S, Gaeta L, et al. Should oncologists be aware in their clinical practice of KRAS molecular analysis? J Clin Oncol 2011;29: e206-e207.

46 Benhaim L, Maley K, Le Corre D, et al. Reply to D. Santini et al. J Clin Oncol 2011;29:e208-e209.

47 Ogino S, Kawasaki T, Brahmandam M, et al. Sensitive sequencing method for KRAS mutation detection by Pyrosequencing. J Mol Diagn 2005;7:413-421.

48 Thomas RK, Nickerson E, Simons JF, et al. Sensitive mutation detection in heterogeneous cancer specimens by massively parallel picoliter reactor sequencing. Nat Med 2006;12:852-855. 